\title{
Orthopaedic applications of nanoparticle-based stem cell therapies
}

\author{
Ian Wimpenny, Hareklea Markides and Alicia J El Haj*
}

\begin{abstract}
Stem cells have tremendous applications in the field of regenerative medicine and tissue engineering. These are pioneering fields that aim to create new treatments for disease that currently have limited therapies or cures. A particularly popular avenue of research has been the regeneration of bone and cartilage to combat various orthopaedic diseases. Magnetic nanoparticles (MNPs) have been applied to aid the development and translation of these therapies from research to the clinic. This review highlights contemporary research for the applications of iron-oxide-based MNPs for the therapeutic implementation of stem cells in orthopaedics. These MNPs comprise of an iron oxide core, coated with a choice of biological polymers that can facilitate the uptake of MNPs by cells through improving endocytic activity. The combined use of these oxides and the biological polymer coatings meet biological requirements, effectively encouraging the use of MNPs in regenerative medicine. The association of MNPs with stem cells can be achieved via the process of endocytosis resulting in the internalisation of these particles or the attachment to cell surface receptors. This allows for the investigation of migratory patterns through various tracking studies, the targeting of particle-labelled cells to desired locations via the application of an external magnetic field and, finally, for activation stem cells to initiate various cellular responses to induce the differentiation. Characterisation of cell localisation and associated tissue regeneration can therefore be enhanced, particularly for in vivo applications. MNPs have been shown to have the potential to stimulate differentiation of stem cells for orthopaedic applications, without limiting proliferation. However, careful consideration of the use of active agents associated with the MNP is suggested, for differentiation towards specific lineages. This review aims to broaden the knowledge of current applications, paving the way to translate the in vitro and in vivo work into further orthopaedic clinical studies.
\end{abstract}

\section{Introduction}

Stem cells have an ever-increasing number of applications in the field of regenerative medicine. They can now be used to treat many diseases or conditions by replacing and restoring the function of cells, tissues or organs in order to establish normal function [1]. The sources of stem cells further extend the number of therapeutic applications; this is combined with stem cell research, steadily moving from the bench-top towards clinical applications. Stem cells have the ability to migrate within tissues and on substrates, which is often guided by the presence of chemical mediators and topography [2]. The migration of these cells to and from the target location requires monitoring to determine the effectiveness of the

\footnotetext{
*Correspondence: a.j.el.haj@bemp.keele.ac.uk
}

Institute of Science and Technology in Medicine, Keele University, The Guy Hilton Research Centre, Thornburrow Drive, Hartshill, Stoke-on-Trent, Staffordshire ST4 7QB, UK therapy. It is highly desirable to have a multifunctional mechanism for targeting, tracking and stimulating stem cells both in vivo and in vitro; this can be achieved through the use of nanoparticles [3]. The use of nanoparticles can provide answers to questions such as: What is the optimal delivery route? What is the extent of engraftment? What are the migratory patterns post transplantation? What is the ideal dosage scheme? Having the answer to these questions will help in the optimisation of the overall therapy and thus in increasing its therapeutic potential [4]. A technique that is capable of providing these answers would be very important to the field of tissue engineering; the use of magnetic nanoparticles (MNPs) is potentially a suitable method.

In a broad context, we see that the application of nanotechnology within the remit of stem cell therapeutics is increasing. Research has already been undertaken using various nanoscale materials, including nanoparticles (both metallic/magnetic and ceramic), nanofibres and carbon nanotubes, amongst others [5]. The major benefit of using nanoparticles is that, due to their 
size, they have the unique ability to be in close proximity to a specific biological entity or marker [6] and interact with it on a cellular (10 to $100 \mu \mathrm{m}$ ), subcellular (20 to $250 \mathrm{~nm})$, protein $(3$ to $50 \mathrm{~nm})$ or genetic $(10$ to $100 \mathrm{~nm}$ ) scale $[7,8]$. MNPs specifically have a multifunctional aspect within this field, where they can be used in the tagging, tracking and activation of stem cells both in vitro and in vivo. This multifunctional propensity with relation to orthopaedic therapies will be the main focus of the present review.

\section{Magnetic nanoparticles}

MNPs have traditionally been used for a number of biomedical applications, including cell separation, automated DNA extraction, gene targeting, drug delivery, diagnostics and hyperthermia $[9,10]$. However, there are far more diverse applications, as shown by their employment in the field of regenerative medicine; for example, cell transfection and magnetic-force stimulation of cells [11].

In general, nanoparticles can be generated by a number of methods, depending on the material to be used, but include co-precipitation, microemulsions, thermal decomposition, metal-reducing bacteria and the use of polyols $[6,9,10]$, and are manufactured from a range of synthetic or biological materials - for example, liposomes, polymer, protein, dendrimers, biodegradable polymer nanoparticles and carbon-based nanoparticles, and MNPs [12]. A number of metals such as nickel, cobalt and iron can be used to convey the magnetic properties of these MNPs. The behaviour of the MNP within an applied magnetic field depends on a number of factors directly related to the properties of the MNP, such as size [13] and magnetisation [14]. When considering MNPs for biomedical application, the toxic nature of the particles must also be investigated thoroughly and clinical trials have to be performed to further assess this aspect [15]. It has been found that certain metals, cobalt and nickel for example, are toxic to biological entities and therefore not suitable for biomedical applications, while others such as iron-oxide-based particles are considered safer $[16,17]$.

Magnetic materials are commonly classified according to their susceptibility to magnetic fields. Paramagnetic substances are only magnetised when exposed to a magnetic field, while ferromagnetic materials remain magnetised even after the magnetic field has been removed [16]. Importantly, ferromagnetic materials develop paramagnetic properties as they decrease in size to approximately $20 \mathrm{~nm}$ in diameter, or $3 \mathrm{~nm}$ in the case of pure iron beads $[6,18]$. This implies that once the magnetic field has been removed the particles are no longer magnetic while still maintaining a higher saturation magnetism than paramagnetic materials, and so are named superparamagnetic. For the biomedical application of MNPs, particles exhibiting superparamagnetic properties are preferred as this implies that the particles will not be attracted to each other and so the risk of agglomeration in a medical setting is minimised [19]. In addition, the biocompatible and low-toxic nature of the particles is highly advantageous [17]. Particles possessing these properties are known as superparamagnetic iron oxide nanoparticles (SPIONs).

As previously mentioned, MNPs can be coated with organic/inorganic polymers to improve their biocompatibility. These include proteins or ligands such as RGD, fibronectin, collagen type I, poly-L-lysine and dextran [10]. Surface coatings can be customised and designed according to specific needs by altering surface charges, protein binding capacity, surface topography and biocompatibility [20]. This further functionalises the particles, allowing them to interact with the cell for tagging and targeting of biological entities with minimal toxic effects $[7,21]$.

\section{Iron oxide nanoparticles}

Iron oxide MNPs can be categorised by size: ultra-small SPIONs, which are approximately 10 to $40 \mathrm{~nm}$ in diameter [22]; monocrystalline iron oxide nanoparticles, which are a subcategory of ultra-small SPIONs, characterised by the crystalline state of their core (typically 10 to $30 \mathrm{~nm}$ in diameter) [23]; SPIONs, approximately 50 to $200 \mathrm{~nm}$ in diameter; and, finally, microparamagnetic iron oxide particles, which are approximately $300 \mathrm{~nm}$ to $3.5 \mu \mathrm{m}$ in diameter $[24,25]$. SPIONs are typically composed of either a magnetite $\left(\mathrm{Fe}_{3} \mathrm{O}_{4}\right)$ or maghemite $\left(\gamma-\mathrm{Fe}_{2} \mathrm{O}_{3}\right)$ core [26] coated with an organic/inorganic polymer [27] or precipitated throughout a porous biocompatible polymer [14].

The precedent for using SPIONs in a biological setting comes from the application of SPION-based magnetic resonance imaging (MRI) contrast agents [16]. It is thought that these contrast agents can be used for a variety of purposes in the clinical translation of stemcell-based therapies for the tagging, tracking and activation of stem cells and other cell lines [28,29]. Feridex (Berlex Biosciences, Richmond, VA, USA), or Endorem in Europe (Guerbet, Sulzbach, Germany), are examples of FDA-approved, iron-based MRI contrast agents, but have unfortunately recently been taken off the market [30].

\section{Importance of the use MNPs in stem cell therapy in orthopaedics}

Traditionally, severe orthopaedic medical conditions have been treated surgically by the insertion of artificial implants or tissue grafts to restore the function of the damaged tissue. However, implant failure, immune rejection, limited supply and donor site morbidity are issues requiring attention. Regenerative medicine offers an 
alternative therapeutic approach to meeting a vast proportion of orthopaedic needs [31]. Mesenchymal stem cells (MSCs) are a multipotent cell source that has shown tremendous promise in the regeneration of connective tissue, including cartilage, bone, tendon and muscle [32]. The combination of stem cells with materials science therefore provides a strong opportunity for therapeutic advances [33]. Regenerative medicine has commonly been used in the therapeutic treatment of osteoarthritis, a disease that affects 8.5 million people in the UK alone. Osteoarthritis is characterised by the destruction of the synovial joint [34]. Surgical techniques range from simple pain management (lavage and debridement) to total knee replacement. However, this therapy is limited by implant failure and is restricted to patients over the age of 60 due to the limited lifespan of the implant [33]. Autologous chondrocyte implantation has therefore been developed, involving isolation, in vitro expansion and subsequent reimplantation of autologous articular chondrocytes into the defect site $[31,33]$. Tissue engineering has also proven promising in the treatment of herniated intervertebral discs with the applications of autologous disc cells [34].

Although these therapies have been successful in the short term, the long-term prognosis of the treatment, although optimistic, is mixed [31,35]. In response to this, it has become necessary to assess the in vivo parameters associated with these therapies, in order to accurately gauge the success of these cellular therapies, particularly in the long term [36]. Evaluation of the short-term and long-term survival of the cells, the rate of repair and the number of cells remaining (or migrating to/from) the desired location (biodistribution) post transplantation is useful in evaluating therapeutic performance [30] but extensive evaluation of efficacy and safety is still to be conducted [12]. There is evidence to suggest that loading of SPIONs with drugs can actually reduce the toxicity of drugs; for example, doxorubicin by association with thermally cross-linked SPIONs (Dox@TCL-SPIONs) [37]. In addition, a recent review highlights that cytotoxicity studies using MNPs or various types and forms (for example, quantum dots) are conducted at concentrations often not relevant to a clinical scenario, and as a result discourage further testing and limit the belief in clinical applicability of the technology [12]. It is noteworthy that heavy metal ions within particles such as quantum dots are the main tributary to cytotoxicity [38].

The biodistribution of cells is dependent on a number of factors; that is, the site of implantation, target organ, mode of delivery and cell type [39]. Ultimately, quantitation of these factors is essential for optimisation of cell delivery methods and dosage schemes [40]. Traditionally, this has been done by carrying out histological and immunohistochemical tests [41-43], which require the termination of an experiment to collect tissue samples for assessment. Long-term follow-up of the therapy is therefore impossible and may require extensive use of animals for in vivo models $[41,44,45]$. This highlights the importance of a non-invasive technique that will contribute to the accurate and reproducible evaluation of the success of therapy $[13,45,46]$. It is thought that a great deal of in vivo information can be gathered through the use of MNP technologies, thus optimising not only these therapies but also the large portfolio of orthopaedic cellbased therapies [46]. The typical schema for the in vivo therapeutic applications overall is indicated in Figure 1; the use of SPIONs in regenerative medicine and tissue engineering purposes will be highlighted.

\section{Labelling of stem cells with SPIONs}

Labelling agents should be biocompatible, nontoxic and should not affect the differentiation state of stem cells or their multipotency/pluripotency; they must have the potential to be detected and monitored; and, finally, they must be versatile enough to be adapted to various cell types, including MSCs [47]. As previously mentioned, the association of SPIONs with stem cells offers an attractive method for tracking, targeting and controlling cells [48]. Broadly speaking, this association is achieved through either the internalisation of SPIONs or the binding of SPIONS to cell surface markers (for example, integrins) or to specific antibodies $[4,49,50]$. Internalisation of SPIONs typically occurs by processes such as endocytosis; that is, the engulfment of the SPIONs into the internal environment of the cells by receptor-mediated responses - for example, adsorptive endocytosis or the use of cell penetrating peptides/proteins (nonspecific attachment) [30]. This method increases the local density of SPIONs [45,51] and is favoured for in vivo applications. The rate of internalisation depends on a number of factors, including cell type, particle size, hydrophobicity, surface charge of the particle polymer and the rate of cell proliferation [20]. Once internalised, within the cell, the SPIONs can be detected and/or manipulated through magnetism to yield a response [6]. It is important to note that through the proliferation of cells, the quantity of SPIONs relative to cell density diminishes during expansion of cells; that is, the dilution effect [30]. Furthermore, exocytosis can potentially remove the SPIONs after internalisation [45]. Internalisation is achieved through the simple incubation of cells with the SPIONs, so evoking spontaneous uptake [28]. The particles are then localised within the endosomes of the cell and fused with lysosomes [30,50]. This method tends to be time consuming and is limited to cells that have a high degree of phagocytosis [52]. Figure 2 illustrates the mechanisms for the internalisation of SPIONs.

Cells exhibiting a low degree of phagocytosis may require prolonged incubation for efficient internalisation 


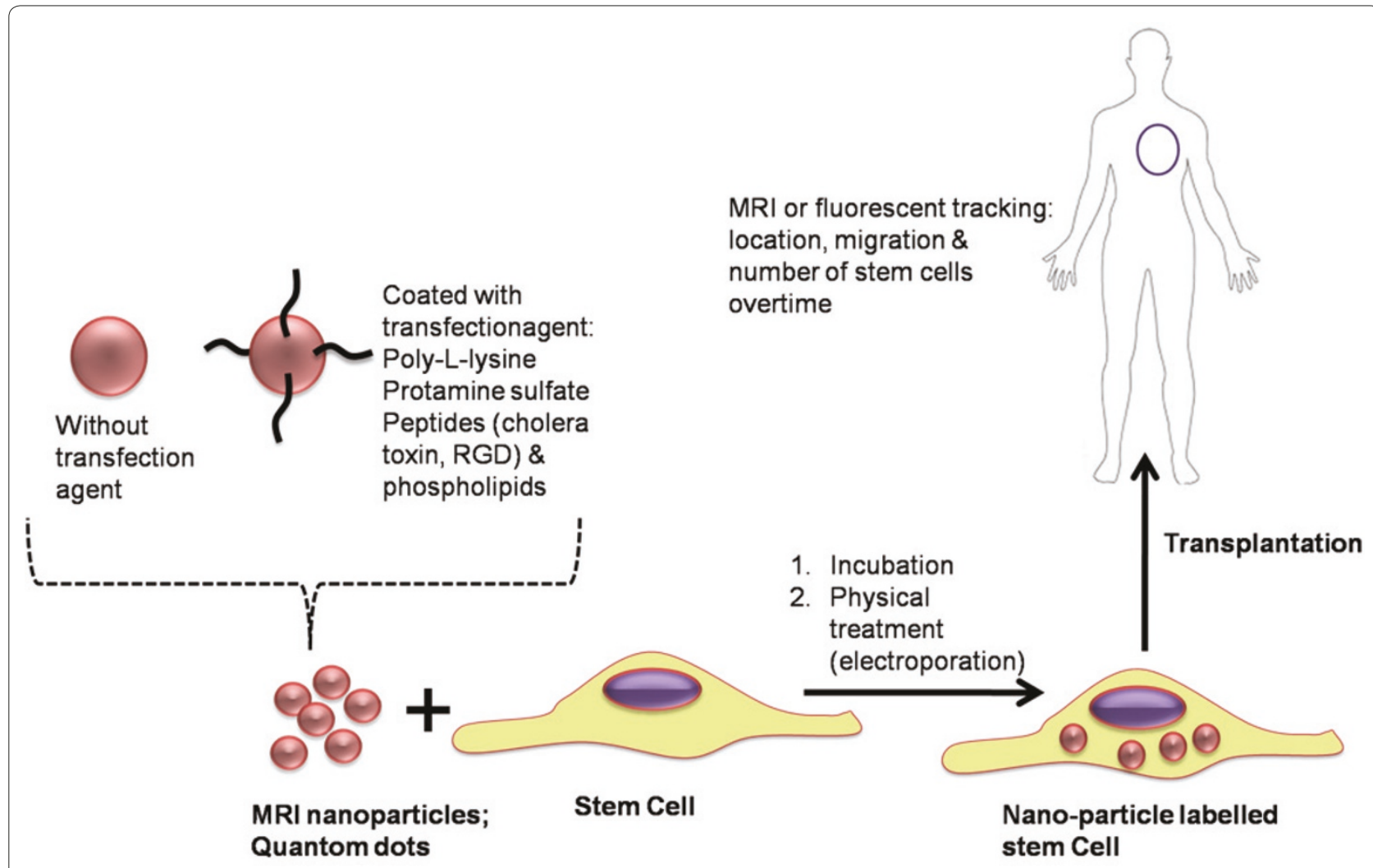

Figure 1. In vivo therapeutic applications of magnetic nanoparticles. Schematic diagram for the creation and functionalisation of the magnetic nanoparticles and the internalisation and implantation into stem cell labelling for magnetic resonance imaging (MRI) and fluorescence tracking.

of SPIONs. This may be undesirable, as extended culture could impair the potency of the cells or redirect differentiation towards an unwanted lineage [53]. The use of transfection agents can be employed to increase labelling efficiency in a shorter period of time, as the agents can form complexes with superparamagnetic iron oxide, increasing uptake by phagocytosis [21,28]. Examples include poly-L-lysine, protamine and cationic liposomes [52]. Kostura and colleagues found that Feridex poly-Llysine in fact inhibited chondrogenesis, yet this was not found with Feridex protamine [54,55]. Therefore, the effect of the transfection agent must be investigated further as it may interfere with the activities of the cell [56]. To overcome the limitations incurred by internalisation and the use of transfection agents, a technique known as magnetoelectroporation has been introduced [30]. Essentially, a magnetic field is used to induce the accumulation of the contrast agent in the cytoplasm of the cell by increasing membrane permeability [52]. This eliminates the need for transfection agent antibodies, peptides and cell culturing all together [30], but unfortunately the method is considered somewhat damaging to cells. Previous studies have shown that gene transfection can be improved using techniques such as magnetofection, which utilises the stimulation of MNPs (for example, SPIONs) by a magnetic field to enhance the transfection efficiency of associated genes. This has been demonstrated to be effective both in vitro and in vivo $[57,58]$.

Previous investigations have assessed the effect of labelling cells with SPIONs on the differentiation of stem cells [49]. The majority of these investigations report that SPIONs are frequently nontoxic to stem cells and do not affect the differentiation [54,59], proliferation, metabolic expression profile, reactive oxygen species formation and apoptosis rate [60]. A recent report by Balakumaran and colleagues using SPIONs and different contrast agents, including ferumoxides, indicates that bone-marrowderived MSCs could retain their multipotentiality and could differentiate to adipogenic, chondrogenic and osteogenic lineages [61]. This provides strong hope for the future of tracking and targeting stem cells in vivo to assess therapeutic applications.

\section{Tracking, targeting and activation of SPION-labelled cells}

The use of SPIONs in regenerative medicine is beneficial because they are multifunctional and can be used for the 


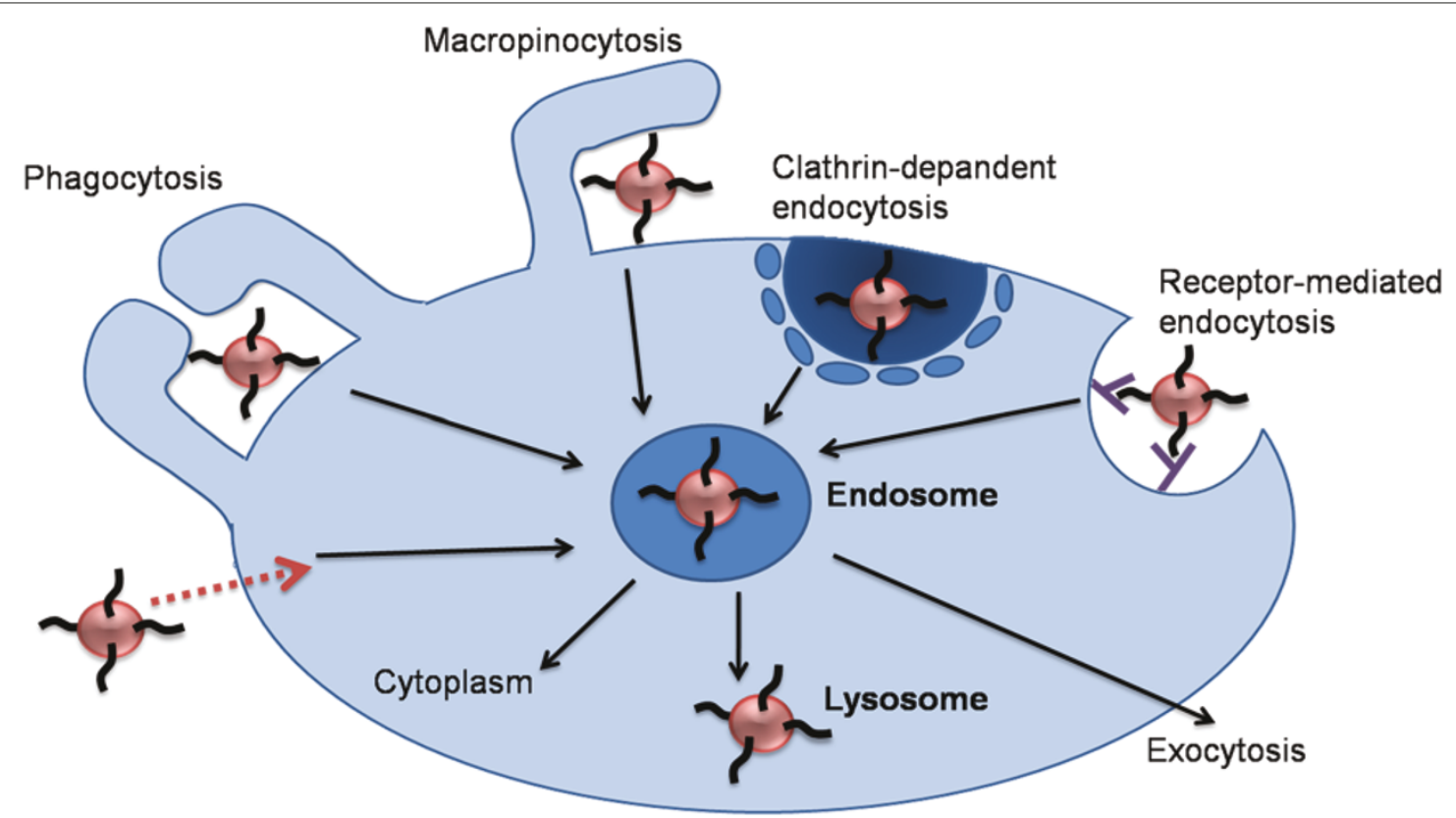

Figure 2. Mechanisms for the internalisation of superparamagnetic iron oxide nanoparticles. Schematic diagram for the different cellular uptake mechanisms of coated magnetic nanoparticles. Adapted from [45,92].

tracking, targeting and activation of stem cells both in vivo and in vitro. These aspects are now explored in greater detail.

\section{Tracking}

It is very difficult to find the ideal combination of labelling agent and corresponding imaging tool that can successfully meet clinical needs. There are many noninvasive biological imaging devices commercially available, none of which are completely suited for the monitoring and tracking of magnetically labelled stem cells to the accuracy and specificity needed. These include MRI and magnetic resonance spectroscopy, which suffer from low sensitivity but have high resolution (that is, 25 to $100 \mu \mathrm{m}$ ) [62]. Alternatively, positron emission tomography and bioluminescence imaging have higher sensitivity but lower resolution (that is, 1 to $2 \mathrm{~mm}$ ) [62]. MRI is traditionally linked to the use of FDA-approved SPIONbased contrast agents and is typically the most applicable medical imaging tool for this specific application because it is available in a vast majority of medical institutions [42]. This implies that the overall system is safe and thus requires little manipulation when used in regenerative medicine

\section{Magnetic resonance imaging for tracking of SPION-loaded cells}

Successful detection of SPION-labelled cells depends on the number of cells implanted, accumulation of iron in cells, spatial resolution of the image, the field strength of MRI, the signal:noise ratio and the pulse sequence $[30,51]$. Detection of SPIONs is attributed to the disruption of the magnetic field by the iron core. This disruption can be translated into a loss of signal and can be visualised as a hypointense region on T2 MRI sequences [48]. MRI has a resolution of about 25 to $100 \mu \mathrm{m}$ resolution [62], which permits tracking of individual cells while still monitoring the surrounding anatomical structures. This ability is particularly useful for the clinician as information on the pathology of the surrounding tissue is a major issue when assessing the success of the therapy [4]. Furthermore, MRI allows for real-time correlation of cell localisation with corresponding, measurable physiological outcomes [53]. Conversely, MRI lacks the ability to determine cell potency or the differentiation state of the cells [51]. To tackle the limitations of traditional MRI techniques, a combination of various techniques has been suggested for tracking cells with SPIONs. The application of bifunctional contrast agents is interesting as it allows multimodal imaging [42]; for example, incorporating an organic, fluorescent dye into a silica-coated shell of a particle with a magnetite core [50]. This bifunctionality offers the combined benefits of an iron-based contrast with the greater sensitivity of a fluorescent dye [40]. An example of one such agent is the multimodal nanoparticle MNPs@SiO, (FITC), which has been shown to be a biocompatible contrast agent successful in the labelling of MSC [47].

This use of MRI has shown great potential for in vitro studies [63], but currently the translation for the use of fluorescent dyes in clinical applications is limited as they cannot be detected in vivo. This clinical use may be 


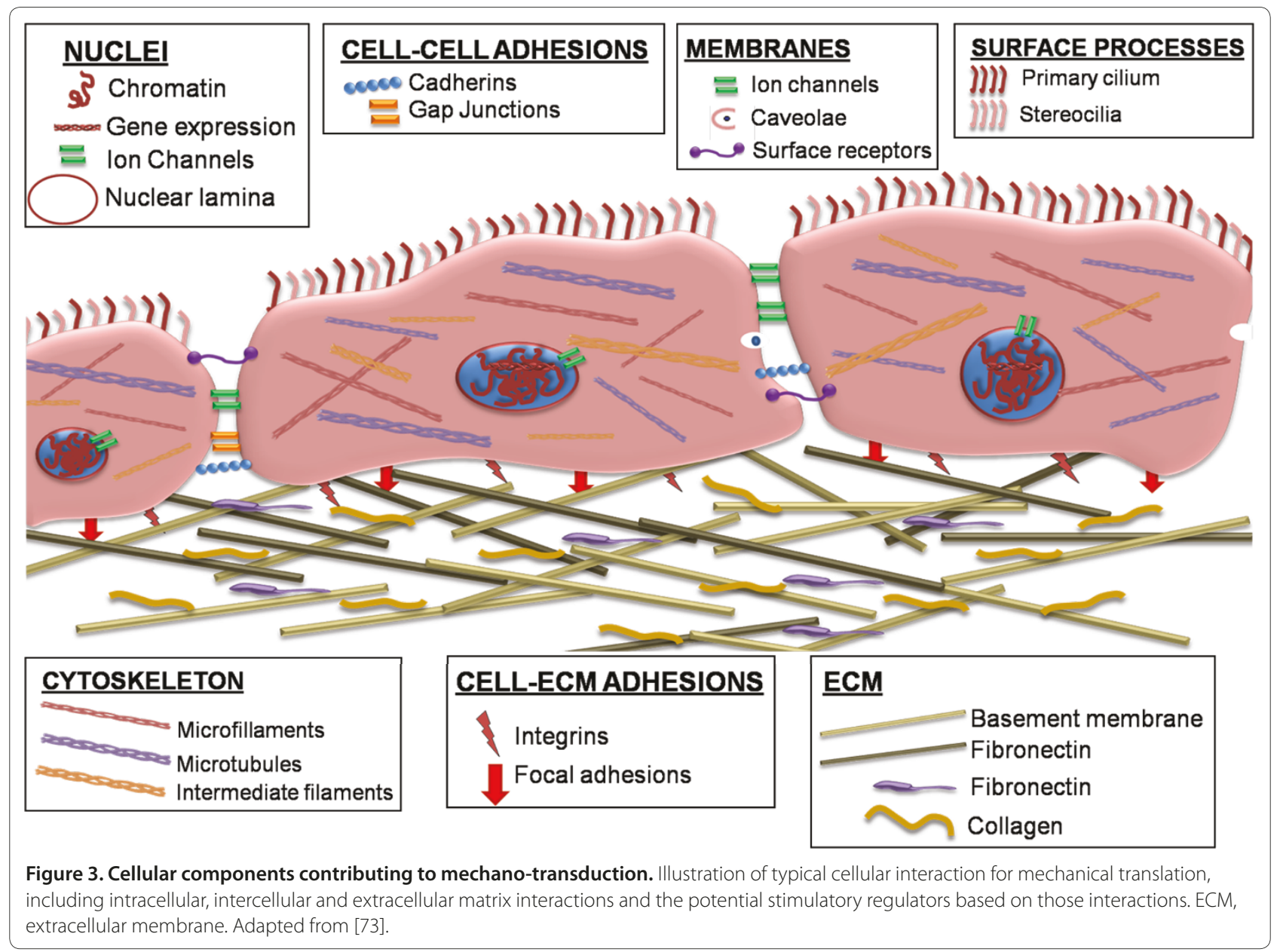

possible in the near future, however, as iron oxide particles could be detected by MRI and the identification of fluorescent markers; for example, green fluorescent protein could be detected by near-infrared fluorescence imaging in vivo [64]. Furthermore, applying a combination of imaging techniques such as positron emission tomography, single-photon emission computed tomography, ultrasound and optical imaging in conjunction with MRI may also provide more details regarding the behaviour of cells, while offering excellent anatomical resolution [43] for advanced analysis of therapeutic effect [59].

\section{Targeting of magnetically labelled stem cells}

Incorporating SPIONs into cells allows noncontact manipulation of cells using an external magnetic field gradient. This can be used to precisely position or target the cells at the site for regeneration or repair $[65,66]$, which is a powerful, non-invasive tool in stem cell therapy $[67,68]$. Importantly for orthopaedics, this technology has been applied in the vascularisation of various tissues by endothelial progenitor cells. Endothelial progenitor cells have a negatively charged surface, so they are capable of nonspecific binding to anionic magnetic particles. Attaching magnetic particles to these cells does not affect differentiation or proliferation of the cells and also does not alter the membrane proteins that are essential for vascularisation [69]. Attaching magnetic particles to endothelial progenitor cells is thought to help in localised cell-based therapies as vascularisation is essential for the formation and development of many tissues. Directing these cells from a distance with an external magnetic field to areas that require vascularisation can thus enhance the regeneration/repair for damaged tissues. The targeting of cells to a specific site and monitoring their behaviour in a model system can therefore be examined and translated to in vivo studies [67]. This has recently been achieved by our group [70], whereby MSCs labelled with SPIONs could be trapped in a fluid flow vessel in vitro, indicating that site-specific targeting is possible. The positive data, based on a model system, led to an investigation for the implantation of a magnet subcutaneously to determine the potential for trapping and targeting labelled MSCs to a specific location. We confirmed that site-specific targeting was 


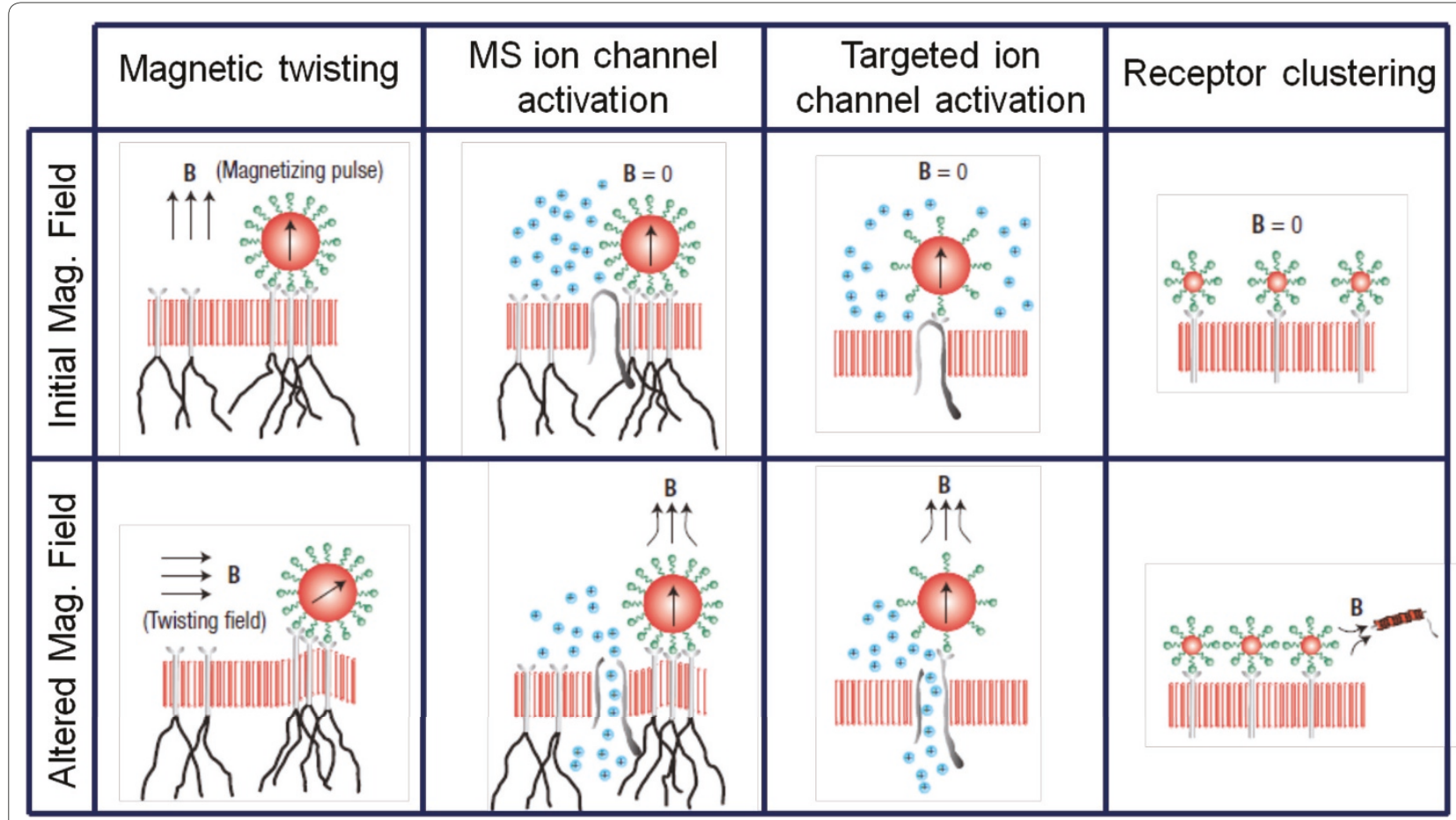

Figure 4. Different mechanisms for mechanical stimulation using magnetic particles. Magnetic twisting cytometry: actin filaments are stimulated by the attachment of large $(1 \mu \mathrm{m})$ magnetic nanoparticles (MNPs) to integrin receptors on the cell membrane. A magnetic pulse is applied, followed by a torque being applied to the particle, resulting in a twisting motion. Mechano-sensitive (MS) ion channel activation: large MNPs are attached to the integrin. The magnetic force causes the particle to be pulled towards the field, thus deforming the membrane and activating the adjacent MS ion channel. Targeted ion channel activation: MNPs (100 nm to $2.7 \mu \mathrm{m}$ ) are attached to certain MS ion channels via corresponding antibodies. Applying a magnetic force forces the channel to open. Receptor clustering: MNPs (30 nm) are bound to receptor complexes along the membrane, and once a force is applied with a magnetic needle the receptors are pulled towards the field source, thus initiating receptor clustering. Adapted from [3].

possible in vivo (manuscript submitted for publication). This finding is highly promising, because once cells can be targeted to a specific location the SPIONs bound to specific cell membrane molecules (for example, ion channels or ligands) can be activated and manipulated.

\section{Activation of stem cells using SPIONs}

Mechanical stimulation can be used to facilitate cell differentiation, proliferation and migration of stem cells [71]; physical forces include fluid flow, axial compression, tension and magnetism [72]. This stimulation is achieved through mechano-transduction, which is the process whereby cells can convert physiological mechanical stimuli into biochemical signals [73]. Mechano-transduction can be subdivided into three separate areas: mechanocoupling (mechanical trigger), cell-cell communication (distribution of the mechanical stimuli throughout a tissue) and the effector response (that is, the response to that message - for example, remodelling of the tissue) [74]. Attaching SPIONS to specific cell surface receptors can induce various effects when an external magnetic field is applied - for example, membrane stiffening, changes in the cell cytoskeleton (stiffening of actin filaments), $\mathrm{Ca}^{2+}$ influx into the cells, mRNA and ribosome moving to focal adhesion complexes, increase in intracellular cAMP, increase in CREB phosphorylation, increase in regulation in endothelin-1 expression and downstream activation of ion channels via FAK kinase $[20,75]$. Other changes also include regulation of mitogen-activated protein kinase activity, change in intracellular $\mathrm{pH}$ and increase in tyrosine phosphoregulation [72]. Furthermore, mechanical deformation of the cell membrane can directly activate mechano-sensitive ion channels. This has been shown to significantly affect cell activity, enhancing the regenerative capabilities [20]. A variety of cellular components have been shown to contribute to mechano-transduction [73], as highlighted in Figure 3. Different receptors activate different pathways, but microscopic and genetic techniques cannot identify which receptor initiates a cellular response during mechanostimulation. Selective targeting of receptors with MNPs circumvents the issues associated with macroscopic changes. Figure 4 highlights some of the ways to initiate a cellular response magnetically. 

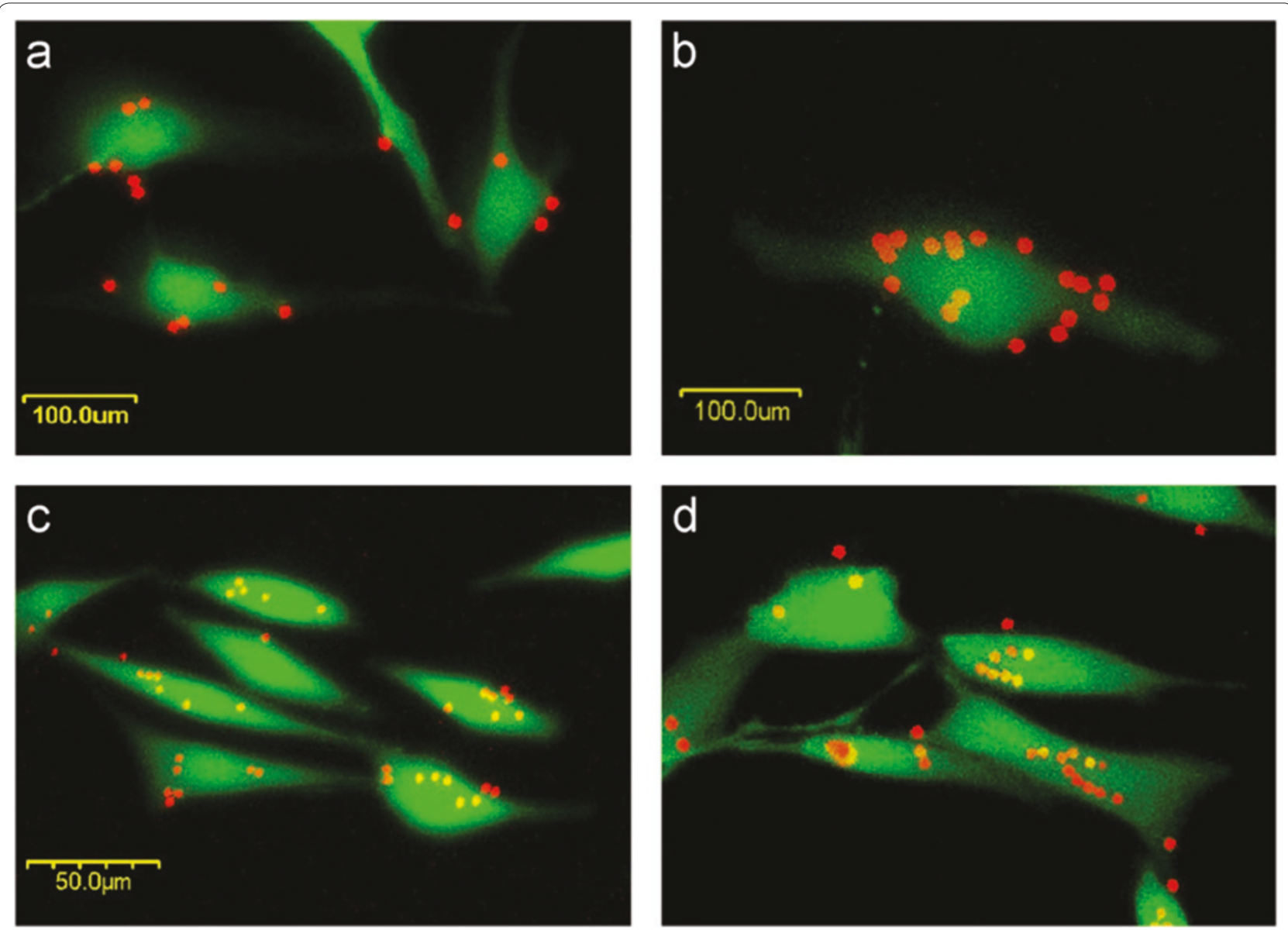

Figure 5. Superparamagnetic iron oxide nanoparticles bind to RGD receptors on the surface of osteoblasts. Confocal laser scanning microscopy images of MG63 bone-like cells after (a), (b) 4 hours and (c), (d) 24 hours incubation with RGD-coated $2.7 \mu$ m 'Flash red'fluorescent magnetic particles. The cytoplasm of viable cells is labelled with Calcein-AM (green). Reprinted from [85].

\section{Clinical application of SPIONs in orthopaedics}

Studies whereby SPIONs have been incorporated into various stem cell populations have yielded some interesting data [30], especially with regard to animal models $[4,76,77]$. Initial proof of concept was demonstrated in the early 1990s when immune cells were labelled with SPIONs. This gave confidence that the concept could be used for a range of cells and that cell migration could be monitored [78]. One universal issue is the inability to obtain large cell numbers for clinical applications. However, the use of magnetite cationic liposomes may be applied to overcome this [79]. Furthermore, this was used to create three-dimensional tissues without the need for scaffold implantation. Human MSCs were labelled with $20 \mathrm{pg} /$ cell magnetite cationic liposomes (this concentration was shown not to affect differentiation towards mesenchymal lineages). The cells were then placed in an ultralow attachment plate. A magnet was placed on the underside of the plate. This caused the cells to aggregate, forming a sheet. Cell sheets were then implanted into a cranial defect of female F344 rats. After 14 days, new bone formation was observed within the defect using the transplanted cell sheets. This was not the case with the control group; no bone formation was observed [80]. In a tracking study, the biodistribution of cells was assessed by labelling the cells with MNPs and monitoring their activity using MRI. Ex vivo studies were carried out with cartilage to determine the ability to track cells and differentiate from surrounding cartilage. Inserting 1,000 cells into a $4 \mathrm{~mm}$ defect could not be detected. When 10,000 and 100,000 cells were implanted, however, clear signals were observed and easily distinguishable from the surrounding cartilage [81]. There are frequent examples of MNPs being used for bone tissue engineering $[82,83]$. MSCs were injected into a joint cavity to investigate the mechanism of cartilage repair with MSCs and to investigate the internal environment of the cavity and how this influences differentiation of certain lineages of MSC. With time, cells were found to have migrated to the marrow sinuses and the synovial fluid. In addition, cells 


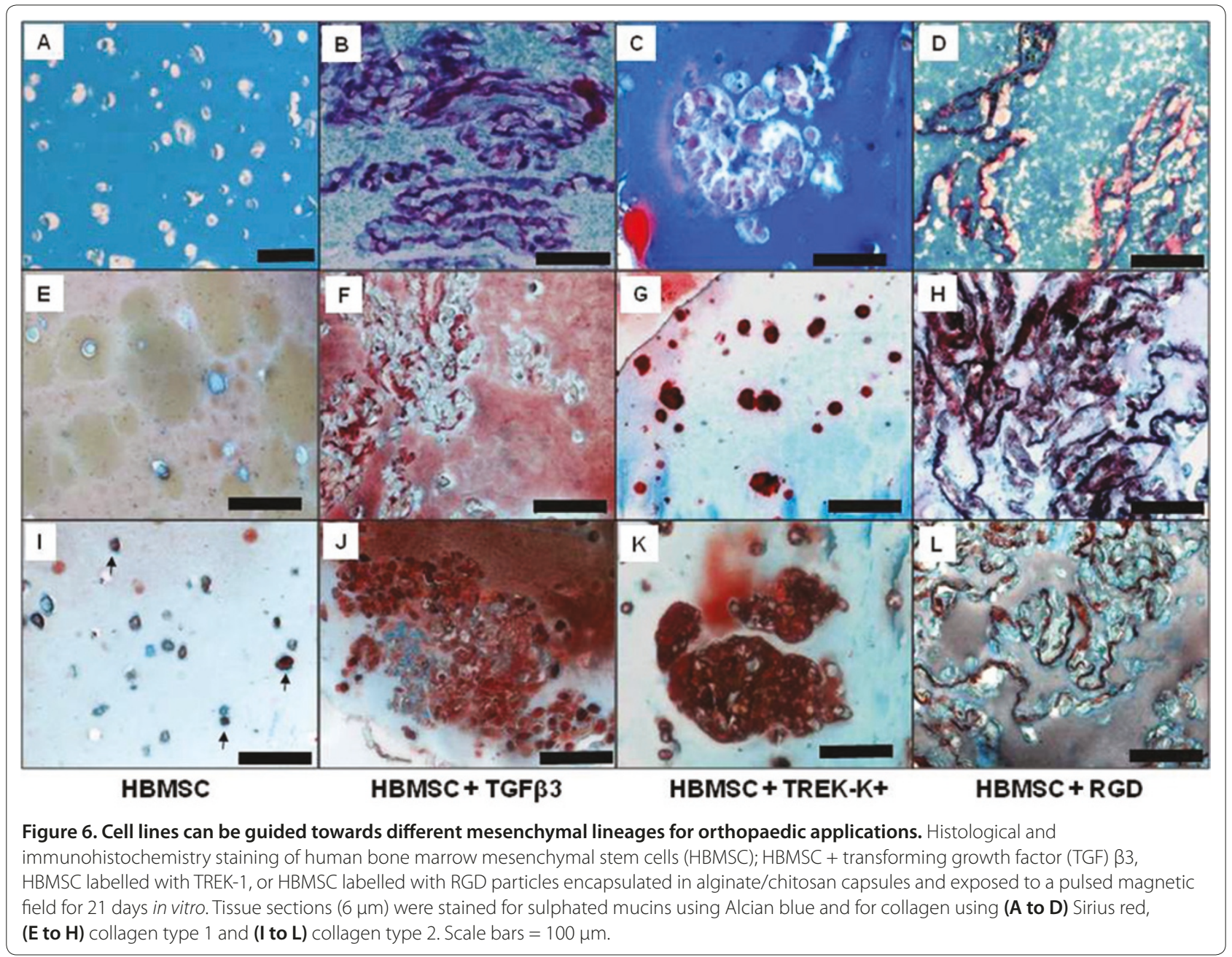

did not migrate to the adjacent cartilage defects, perhaps due to strong collagen networks and the proteoglycan matrix [41].

In our laboratory we have demonstrated how SPIONs can be bound to RGD receptors on the surface of osteoblasts, enabling stimulation over a 3-week period (Figure 5). Following cyclical magnetic stimulation, labelled cells demonstrated upregulation of osteopontin (a key component in the differentiation and activity of the bone cells and the maturation and mineralisation of the bone matrix), and increased osteo-related protein production [72]. These results indicate that culture of SPIONs with osteoblasts does not inhibit osteogenic behaviour. Furthermore, SPIONs can be bound to selected membrane receptors and then subjected to oscillating magnetic fields via a magnetic force bioreactor (MFB) in order to stimulate tagged receptors and provide mechano-transduction through the cell membrane. Many cell-based therapies could benefit from this technology; however, the mechanical conditioning of the cells and the tissues in a bioreactor is a vital part in determining the tissues being produced. The MFB has been used in the production of bone and cartilage. MFBs have a particular advantage over traditional bioreactors in the sense that the MFB decreases the risk of infections as the reactor is operating within a closed system. The cells remain within the container in the specific culture medium and the magnetic field is applied externally; this serves a dual purpose as the supply of nutrients to the cells is limited and not continuous in conventional bioreactors, which can be detrimental in the viability of a stem cell. Cells remain in the container, within a specific culture medium, while an external magnetic force is applied. The supply of nutrients in this system is controlled, not continuously flowing. This eliminates sterility issues and also reduces the application of forces (for example, fluid shear stress), other than magnetic stimulation.. Secondly, the MFB provides a more spatial scalable system, making it the more attractive choice for tissue formation that is not possible with conventional systems. Furthermore, it is thought that in the MFB the mechanical stresses applied can be controlled by altering the strength of the 
magnet, the duration of the force applied and the distance from the cells. Lastly, the stress is applied to the cell membrane; there would be no need to ensure that the cells attach to a mechanically durable scaffold.

Over recent years, our group has performed many investigations into the use of MFBs to stimulate stem cell differentiation [84-89]. Mechanical force is known to have a direct effect on the stimulation of stem cells. Furthermore, it was determined that specific magnetic mechano-conditioning is required during stages of osteogenic differentiation [88]. These studies indicate that MNPs are useful for determining and stimulating specific pathways related to the differentiation of stem cells.

Remote differentiation of stem cells is an exciting potential for magnetic particle technology in the field of tissue engineering, for multiple cell lineages [90]. Stem cell differentiation in target repair tissues can be controlled in vivo through external cycling magnetic fields. It is possible to attach MNPs to specific ion channels; that is, TREK-1. Markers of osteogenic and chondrogenic differentiation were investigated after activation of this channel. The expression of collagen type I, SOX9, RUNX2 and osteopontin mRNA increased, relative to the control samples. Furthermore, both in vitro and in vivo studies confirmed that expression of collagen types I and II and proteoglycan and collagen synthesis could be considerably enhanced using MNPs coated with RGD peptides [83]. These results are therefore a strong indication that various cell lines, including MSCs, can be guided towards different mesenchymal lineages for orthopaedic applications (Figure 6) [87].

\section{Conclusion}

In orthopaedics, cell therapies are moving rapidly forward for routine use in clinical therapies [12]. Defining the success of these therapies and identifying ways to improve and routinely replicate these clinical practices are required to enable further development for the clinic, as MNPs can provide information regarding the optimal route, dosage scheme and localisation and migration of cells for therapeutic treatments. The applications of MNPs in regenerative medicine are diverse, offering a potential route to improve the way in which we can control, monitor and target stem cells to the sites of repair. A large number of in vitro studies have reported that iron-oxide-based MNPs do not affect the viability, proliferation and differentiation capacity of cells in vitro. However, long-term in vivo studies have not been studied as extensively. Successful integration of these techniques into surgical practice relies on building confidence in surgeons that nanoparticles are not harmful and can be used routinely in patients. Stem-cell-based therapies differ from conventional therapies (including pharmaceuticals) in the sense that the cells are the active therapy and must remain in the body and integrate into the in vivo environment to have a therapeutic effect. Research demonstrating the long-term stability and functional, therapeutic repair - both in vitro and in vivo - of the regulatory bodies is an exciting prospect and would pave the way for future clinical applications of nanoparticlebased stem cell therapies [91]. The use of magnetic force, in the context of bioreactors, has proven to be an interesting area, permitting stimulation of targeted surface receptors (for example, ion channels). This broadens the scope for the use of MNPs in orthopaedic applications using stem cells, with particular regard to controlling differentiation. There is hope that the technique will make the translation from bench to bedside in the future. Clinical trials have shown no adverse side effect of labelling stem cells with SPIONs. It is essential to confirm the safety of the procedure prior to in vivo transplantation and, despite clinical efficacy for the technique, long-term evaluation of the safety of using MNPs is yet to be conducted $[12,92]$.

\section{Abbreviations}

MFB, magnetic force bioreactor; MNP, magnetic nanoparticle; MRI, magnetic resonance imaging; MSC, mesenchymal stem cell; SPION, superparamagnetic iron oxide nanoparticle.

\section{Competing interests}

The authors declare that they have no competing interests.

\section{Acknowledgements}

The authors would like to thank SL Wilson and S Hughes for their contributions to this work. Support was received from Expertissues (NMP3-CT-2004-500283) and from the EPSRC Doctoral Training Centre (EP/F/500491/1).

Published: 19 April 2012

\section{References}

1. Mason C, Dunnill P: A brief definition of regenerative medicine. Regen Med 2008, 3:1-5.

2. Hoehn M, Kustermann E, Blunk J, Wiedermann D, Trapp T, Wecker S, Focking M, Arnold H, Hescheler J, Fleischmann BK, Schwindt W, Buhrle C: Monitoring of implanted stem cell migration in vivo: a highly resolved in vivo magnetic resonance imaging investigation of experimental stroke in rat. Proc Natl Acad Sci U S A 2002, 99:16267-16272.

3. Dobson J: Remote control of cellular behaviour with magnetic nanoparticles. Nat Nanotechnol 2008, 3:139-143.

4. Berman SMC, Walczak P, Bulte JWM: Tracking stem cells using magnetic nanoparticles. Wiley Interdiscip Rev Nanomed Nanobiotechnol 2011, 3:343-355.

5. Riehemann K, Schneider SW, Luger TA, Godin B, Ferrari M, Fuchs H: Nanomedicine - challenge and perspectives. Angew Chem Int Ed Engl 2009, 48:872-897.

6. Krishnan KM: Biomedical nanomagnetics: a spin through possibilities in imaging, diagnostics, and therapy. IEEE Trans Magn 2010, 46:2523-2558.

7. Pankhurst QA, Connolly J, Jones SK, Dobson J: Applications of magnetic nanoparticles in biomedicine. J Phys D App/ Phys 2003, 36:R167.

8. Banerjee R, Katsenovich Y, Lagos L, Mclintosh M, Zhang X, Li CZ: Nanomedicine: magnetic nanoparticles and their biomedical applications. Curr Med Chem 2010, 17:3120-3141.

9. Barakat NS: Magnetically modulated nanosystems: a unique drug-delivery platform. Nanomedicine 2009, 4:799-812.

10. Lin M, Kim D, El Haj A, Dobson J: Development of superparamagnetic iron oxide nanoparticles (SPIONS) for translation to clinical applications. IEEE Trans Nanobioscience 2008, 7:298-305.

11. Ito A, Kamihira M: Tissue engineering using magnetite nanoparticles. Prog 
Mol Biol Transl Sci 2011, 104:355-395.

12. Yildirimer $L$, Thanh NTK, Loizidou M, Seifalian AM: Toxicology and clinical potential of nanoparticles. Nano Today 2011, 6:585-607.

13. Ju S, Teng G, Zhang Y, Ma M, Chen F, Ni Y: In vitro labeling and MRI of mesenchymal stem cells from human umbilical cord blood. Magn Reson Imaging 2006, 24:611-617.

14. Mahmoudi M, Sant S, Wang B, Laurent S, Sen T: Superparamagnetic iron oxide nanoparticles (SPIONs): development, surface modification and applications in chemotherapy. Adv Drug Deliv Rev 2011, 63:24-46.

15. Weissleder R, Stark DD, Engelstad BL, Bacon BR, Compton CC, White DL, Jacobs P, Lewis J: Superparamagnetic iron oxide: pharmacokinetics and toxicity. Am J Roentgenol 1989, 152:167-173.

16. Hofmann-Amtenbrink M, Hofmann H, Montet X: Superparamagnetic nanoparticles -a tool for early diagnostics. Swiss Med Wkly 2010, 140:7-13

17. Berry CC: Possible exploitation of magnetic nanoparticle-cell interaction for biomedical applications. J Mater Chem 2005, 15:543-547.

18. Bean CP: Hysteresis loops of mixtures of ferromagnetic micropowders. J Appl Phys 1955, 26:1381-1383.

19. Getzlaff M: Fundamentals of Magnetism. New York: Springer; 2008

20. Hughes S, El Haj AJ, Dobson J: Magnetic micro- and nanoparticle mediated activation of mechanosensitive ion channels. Med Eng Phys 2005, 27:754-762.

21. Solanki A, Kim JD, Lee K-B: Nanotechnology for regenerative medicine: nanomaterials for stem cell imaging. Nanomedicine 2008, 3:567-578

22. Weissleder R, Elizondo G, Wittenberg J, Rabito CA, Bengele HH, Josephson L: Ultrasmall superparamagnetic iron oxide: characterization of a new class of contrast agents for MR imaging. Radiology 1990, 175:489-493.

23. Shen T, Weissleder R, Papisov M, Bogdanov A, Brady TJ: Monocrystalline iron oxide nanocompounds (MION): physicochemical properties. Magn Reson Med 1993, 29:599-604

24. Long CM, Bulte JWM: In vivo tracking of cellular therapeutics using magnetic resonance imaging. Expert Opin Biol Ther 2009, 9:293-306.

25. Thorek D, Chen A, Czupryna J, Tsourkas A: Superparamagnetic iron oxide nanoparticle probes for molecular imaging. Ann Biomed Eng 2006, 34:23-38.

26. Gupta AK, Gupta M: Synthesis and surface engineering of iron oxide nanoparticles for biomedical applications. Biomaterials 2005, 26:3995-4021.

27. Pardoe H, Chua-anusorn W, St Pierre TG, Dobson J: Structural and magnetic properties of nanoscale iron oxide particles synthesized in the presence of dextran or polyvinyl alcohol. J Magn Magn Mater 2001, 225:41-46.

28. Bulte JWM: In vivo MRI cell tracking: clinical studies. Am J Roentgenol 2009, 193:314-325.

29. van Buul GM, Farrell E, Kops N, van Tiel ST, Bos PK, Weinans H, Krestin GP, van Osch GJVM, Bernsen MR: Ferumoxides-protamine sulfate is more effective than ferucarbotran for cell labeling: implications for clinically applicable cell tracking using MRI. Contrast Media Mol Imaging 2009, 4:230-236.

30. Zhou R, Acton PD, Ferrari VA: Imaging stem cells implanted in infarcted myocardium. J Am Coll Cardiol 2006, 48:2094-2106.

31. Oakes BW: Orthopaedic tissue engineering: from laboratory to the clinic. Med J Aust 2004, 180:S35-S38.

32. Caplan Al: Mesenchymal stem cells: cell-based reconstructive therapy in orthopedics. Tissue Eng 2005, 11:1198-1211.

33. Vats A, Tolley NS, Buttery LDK, Polak JM: The stem cell in orthopaedic surgery. J Bone Jt Surg Br 2004, 86B:159-164.

34. Roberts S, Genever P, McCaskie A, De Bari C: Prospects of stem cell therapy in osteoarthritis. Regen Med 2011, 6:351-366.

35. Behrens P, Bitter T, Kurz B, Russlies M: Matrix-associated autologous chondrocyte transplantation/implantation (MACT/MACI) - 5-year followup. Knee 2006, 13:194-202.

36. Tang C, Russell PJ, Martiniello-Wilks R, J. Rasko JE, Khatri A: Concise review: Nanoparticles and cellular carriers - allies in cancer imaging and cellular gene therapy? Stem Cells 2010, 28:1686-1702.

37. Yu MK, Jeong YY, Park J, Park S, Kim JW, Min JJ, Kim K, Jon S: Drug-loaded superparamagnetic iron oxide nanoparticles for combined cancer imaging and therapy in vivo. Angew Chem Int Ed Engl 2008, 47:5362-5365.

38. Derfus AM, Chan WCW, Bhatia SN: Intracellular delivery of quantum dots for live cell labeling and organelle tracking. Adv Mater 2004, 16:961-966.

39. Cheng K, Benten D, Bhargava K, Inada M, Joseph B, Palestro C, Gupta S: Hepatic targeting and biodistribution of human fetal liver stem/ progenitor cells and adult hepatocytes in mice. Hepatology 2009, 50:1194-1203

40. Liu W, Frank JA: Detection and quantification of magnetically labeled cells by cellular MRI. Eur J Radio/ 2009, 70:258-264.

41. Jing XH, Yang L, Duan XJ, Xie B, Chen W, Li Z, Tan HB: In vivo MR imaging tracking of magnetic iron oxide nanoparticle labeled, engineered, autologous bone marrow mesenchymal stem cells following intraarticular injection. Joint Bone Spine 2008, 75:432-438.

42. Himmelreich $U$, Dresselaers $T$ : Cell labeling and tracking for experimental models using magnetic resonance imaging. Methods 2009, 48:112-124.

43. Athiraman $H$, Jiang Q, Ding GL, Zhang L, Zhang ZG, Wang L, Arbab AS, Li Q, Panda S, Ledbetter K, Rad AM, Chopp M: Investigation of relationships between transverse relaxation rate, diffusion coefficient, and labeled cell concentration in ischemic rat brain using MRI. Magn Reson Med 2009, 61:587-594.

44. Tran LA, Krishnamurthy R, Muthupillai R, da Graca Cabreira-Hansen M, Willerson JT, Perin EC, Wilson LJ: Gadonanotubes as magnetic nanolabels for stem cell detection. Biomaterials 2010, 31:9482-9491.

45. Ferreira L: Nanoparticles as tools to study and control stem cells. J Cell Biochem 2009, 108:746-752

46. Wu YL, Ye Q, Foley LM, Hitchens TK, Sato K, Williams JB, Ho C: In situ labeling of immune cells with iron oxide particles: an approach to detect organ rejection by cellular MRI. Proc Natl Acad Sci U S A 2006, 103:1852-1857.

47. Park KS, Tae J, Choi B, Kim YS, Moon C, Kim SH, Lee HS, Kim J, Kim J, Park J, Lee $J \mathrm{H}$, Lee JE, Joh JW, Kim S: Characterization, in vitro cytotoxicity assessment, and in vivo visualization of multimodal, RITC-labeled, silica-coated magnetic nanoparticles for labeling human cord blood-derived mesenchymal stem cells. Nanomedicine 2010, 6:263-276.

48. Engberink RDO, van der Pol SMA, Walczak P, van der Toorn A, Viergever MA, Dijkstra CD, Bulte JWM, de Vries HE, Blezer ELA: Magnetic resonance imaging of monocytes labeled with ultrasmall superparamagnetic particles of iron oxide using magnetoelectroporation in an animal model of multiple sclerosis. Mol Imaging 2010, 9:268-277.

49. Lu CW, Hung Y, Hsiao JK, Yao M, Chung TH, Lin YS, Wu SH, Hsu SC, Liu HM, Mou CY, Yang CS, Huang DM, Chen YC: Bifunctional magnetic silica nanoparticles for highly efficient human stem cell labeling. Nano Lett 2006, 7:149-154

50. Sung C, Hong K, Lin S, Lee Y, Cha J, Lee J, Hong C, Han B, Jung S, Kim S, Yoon $\mathrm{K}$ : Dual-modal nanoprobes for imaging of mesenchymal stem cell transplant by MRI and fluorescence imaging. Korean J Radiol 2009, 10:613-622.

51. Ferreira L, Karp JM, Nobre L, Langer R: New opportunities: the use of nanotechnologies to manipulate and track stem cells. Cell Stem Cell 2008, 3:136-146

52. Dousset V, Tourdias T, Brochet B, Boiziau C, Petry KG: How to trace stem cells for MRI evaluation? J Neurol Sci 2008, 265:122-126

53. Walczak P, Ruiz-Cabello J, Kedziorek DA, Gilad AA, Lin S, Barnett B, Qin L, Levitsky H, Bulte JWM: Magnetoelectroporation: improved labeling of neural stem cells and leukocytes for cellular magnetic resonance imaging using a single FDA-approved agent. Nanomed Nanotechnol Bio/ Med 2006, 2:89-94

54. Arbab AS, Yocum GT, Rad AM, Khakoo AY, Fellowes V, Read EJ, Frank JA: Labeling of cells with ferumoxides - protamine sulfate complexes does not inhibit function or differentiation capacity of hematopoietic or mesenchymal stem cells. NMR Biomed 2005, 18:553-559.

55. Kostura L, Kraitchman DL, Mackay AM, Pittenger MF, Bulte JWM: Feridex labeling of mesenchymal stem cells inhibits chondrogenesis but not adipogenesis or osteogenesis. NMR Biomed 2004, 17:513-517.

56. Yiu HHP, Maple MJ, Lees MR, Palona I, El Haj AJ, Dobson J: Preparation and characterization of iron oxide-silica composite particles using mesoporous SBA-15 silica as template and their internalization into mesenchymal stem cell and human bone cell lines. IEEE Trans Nanobioscience 2010, 9:165-170.

57. Scherer F, Anton M, Schillinger U, Henke J, Bergemann C, Krüger A, Gänsbacher B, Plank C: Magnetofection: enhancing and targeting gene delivery by magnetic force in vitro and in vivo. Gene Ther 2002, 9:102-109.

58. Plank C, Scherer F, Schillinger U, Bergemann C, Anton M: Magnetofection: enhancing and targeting gene delivery with superparamagnetic nanoparticles and magnetic fields. J Liposome Res 2003, 13:29-32.

59. Budde MD, Frank JA: Magnetic tagging of therapeutic cells for MRI. J NuCl Med 2009, 50:171-174.

60. Ito A, Hibino E, Honda H, Hata K-i, Kagami H, Ueda M, Kobayashi T: A new methodology of mesenchymal stem cell expansion using magnetic nanoparticles. Biochem Eng J 2004, 20:119-125. 
61. Balakumaran A, Pawelczyk E, Ren J, Sworder B, Chaudhry A, Sabatino M, Stroncek D, Frank JA, Robey PG: Superparamagnetic iron oxide nanoparticles labeling of bone marrow stromal (mesenchymal) cells does not affect their 'stemness'. PLOS ONE 2010, 5:e11462.

62. Massoud TF, Gambhir SS: Molecular imaging in living subjects: seeing fundamental biological processes in a new light. Genes Dev 2003, 17:545-580.

63. Huh YM, Jun YW, Song HT, Kim S, Choi JS, Lee JH, Yoon S, Kim KS, Shin JS, Suh $J S$, Cheon J: In vivo magnetic resonance detection of cancer by using multifunctional magnetic nanocrystals. J Am Chem Soc 2005, 127:12387-12391

64. Medarova Z, Pham W, Farrar C, Petkova V, Moore A: In vivo imaging of siRNA delivery and silencing in tumors. Nat Med 2007, 13:372-377.

65. Kyrtatos PG, Lehtolainen P, Junemann-Ramirez M, Garcia-Prieto A, Price AN, Martin JF, Gadian DG, Pankhurst QA, Lythgoe MF: Magnetic tagging increases delivery of circulating progenitors in vascular injury. J Am Coll Cardiol Cardiovasc Interv 2009, 2:794-802.

66. Ho VHB, Barcza A, Chen R, Müller KH, Darton NJ, Slater NKH: The precise control of cell labelling with streptavidin paramagnetic particles. Biomaterials 2009, 30:6548-6555.

67. Kim J, Lee H, Kang H-J, Park T: The targeting of endothelial progenitor cells to a specific location within a microfluidic channel using magnetic nanoparticles. Biomed Microdevices 2009, 11:287-296.

68. Hsiao JK, Tai MF, Chu HH, Chen ST, Li H, Lai DM, Hsieh ST, Wang JL, Liu HM: Magnetic nanoparticle labeling of mesenchymal stem cells without transfection agent: cellular behavior and capability of detection with clinical 1.5 T magnetic resonance at the single cell level. Magn Reson Med 2007, 58:717-724.

69. Wilhelm C, Bal L, Smirnov P, Galy-Fauroux I, Clément O, Gazeau F, Emmerich J: Magnetic control of vascular network formation with magnetically labeled endothelial progenitor cells. Biomaterials 2007, 28:3797-3806.

70. Sura HS, Magnay J, Attridge K, Zghoul N, Dobson J, El Haj AJ: Gene expression changes in stem cells following targeted localisation in a flow system using magnetic particle technology. ECM 2008, 16:18.

71. Sniadecki NJ: Minireview: A tiny touch: activation of cell signaling pathways with magnetic nanoparticles. Endocrinology 2010, 151:451-457.

72. Cartmell SH, Dobson J, Verschueren S, El Haj AJ: Development of magnetic particle techniques for long-term culture of bone cells with intermittent mechanical activation. Trans Nanobioscience 2002, 1:92-97.

73. Ingber DE: Cellular mechanotransduction: putting all the pieces together again. FASEB J 2006, 20:811-827.

74. Khan KM, Scott A: Mechanotherapy: how physical therapists' prescription of exercise promotes tissue repair. Br J Sports Med 2009, 43:247-252.

75. Cartmell SH, Dobson J, Verschueren S, Hughes S, El Haj AJ: Mechanical conditioning of bone cells in vitro using magnetic micro particle technology. Eur Cells Mater 2002, 4(Suppl 2):130-131.

76. Bulte JWM, Duncan ID, Frank JA: In vivo magnetic resonance tracking of magnetically labeled cells after transplantation. J Cereb Blood Flow Metab 2002, 22:899-907.

77. Cohen ME, Muja N, Fainstein N, Bulte JWM, Ben-Hur T: Conserved fate and function of ferumoxides-labeled neural precursor cells in vitro and in vivo. J Neurosci Res 2010, 88:936-944.

78. Bulte JW ML, Magin RL, Kamman RL, Hulstaert CE, Go KG, The TH, de Leij L:
Selective MR imaging of labeled human peripheral blood mononuclear cells by liposome mediated incorporation of dextran-magnetite particles. Magn Reson Med 1993, 29:32-37.

79. Shimizu K, Ito A, Honda H: Mag-seeding of rat bone marrow stromal cells into porous hydroxyapatite scaffolds for bone tissue engineering. J Biosci Bioeng 2007, 104:171-177.

80. Shimizu K, Ito A, Yoshida T, Yamada Y, Ueda M, Honda H: Bone tissue engineering with human mesenchymal stem cell sheets constructed using magnetite nanoparticles and magnetic force. J Biomed Mater Res Part B: Appl Biomater 2007, 82B:471-480

81. Farrell E, Wielopolski P, Pavljasevic P, Kops N, Weinans H, Bernsen MR, van Osch GJVM: Cell labelling with superparamagnetic iron oxide has no effect on chondrocyte behaviour. Osteoarthritis Cartilage 2009, 17:961-967.

82. Taylor EN, Webster TJ: Multifunctional magnetic nanoparticles for orthopedic and biofilm infections. Int J Nanotechnol 2010, 8:21-35.

83. Kanczler J, Sura H, Magnay J, Oreffo R, Green D, Dobson J, El Haj AJ: Controlled differentiation of human bone marrow stromal cells using magnetic nanoparticle technology. Tissue Eng Part A 2010, 16:3241-3250.

84. Dobson J, Cartmell S, Keramane A, El Haj A: Principles and design of a novel magnetic force mechanical conditioning bioreactor for tissue engineering, stem cell conditioning, and dynamic in vitro screening. IEEE Trans Nanobioscience 2006, 5:173-177.

85. Hughes S, Dobson J, El Haj AJ: Magnetic targeting of mechanosensors in bone cells for tissue engineering applications. J Biomech 2007, 40:S96-S104

86. Hughes S, El Haj AJ, Dobson J: Magnetic micro- and nanoparticle mediated activation of mechanosensitive ion channels. Med Eng Phys 2005, 27:754-762.

87. Hu B, Yang Y, Dobson JP, El Haj AJ, Bader D: Mechanical conditioning using magnetic nanoparticles bound to PDGF receptors on HBMSCs promotes the smooth muscle alpha actin (SMA) expression. In 8th International Conference on Cell \& Stem Cell Engineering (ICCE). Volume 30. Edited by Magjarevic R. Berlin: Springer; 2011:23-25.

88. Hu B, Yang Y, El Haj AJ: Mechanoreceptor activation via magnetic particle regulates differentiation of human bone marrow stromal cells. Histol Histopathol 2011, 26(Suppl 1):360.

89. Hughes S, McBain S, Dobson J, El Haj AJ: Selective activation of mechanosensitive ion channels using magnetic particles. J R Soc Interface 2008, 5:855-863.

90. Yang L, Zhang L, Webster TJ: Nanobiomaterials: state of the art and future trends. Adv Eng Mater 2011, 13:B197-B217.

91. Rogers WJ, Meyer CH, Kramer CM: Technology insight: in vivo cell tracking by use of MRI. Nat Clin Pract Cardiovasc Med 2006, 3:554-562.

92. van Buul GM, Kotek G, Wielopolski PA, Farrell E, Bos PK, Weinans H, Grohnert AU, Jahr H, Verhaar JAN, Krestin GP, van Osch GJVM, Bernsen MR: Clinically translatable cell tracking and quantification by mri in cartilage repair using superparamagnetic iron oxides. PLOS ONE 2011, 6:e17001.

\section{doi:10.1186/scrt104}

Cite this article as: Wimpenny l, et al:: Orthopaedic applications of nanoparticle-based stem cell therapies. Stem Cell Research \& Therapy 2012 $3: 13$ 